\title{
Valoración de la eficacia de una pauta más lenta y frecuente en la administración de hierro intravenoso en pacientes en hemodiálisis durante un año
}

\author{
Mónica Brazález Tejerina ${ }^{1}$ - Isabel Pérez Arranz² - Emilio González Parra ${ }^{3}$ - Nancy Ma Pose Regueiro ${ }^{4}$ \\ - Ma ${ }^{\mathrm{a}}$ Fe Muñoz Moreno ${ }^{5}$
}

${ }^{1}$ Enfermera, Centro de Diálisis Los Pinos. FRIAT. Valladolid

${ }^{2}$ Médico, Centro de Diálisis Los Pinos. FRIAT. Valladolid

${ }^{3}$ Nefrólogo. Fundación Jiménez Díaz. Madrid

${ }^{4}$ Enfermera. Centro de Diálisis Os Carballos. FRIAT. Vigo

${ }^{5} U$. Apoyo a la Investigación. HCU. Valladolid

\section{Sr. Director:}

Uno de los problemas más frecuentes de los pacientes con enfermedad renal crónica (ERC) en hemodiálisis (HD) es la anemia, tratada habitualmente con factores estimuladores de la eritropoyesis. Esta estimulación requiere una mayor disponibilidad de los depósitos corporales de hierro $(\mathrm{Fe})^{1}$. Para alcanzar los niveles que recomiendan las guías (Ferritina entre 200 y 400ng/ $\mathrm{ml}$ - IST entre 30 y $40 \%$ ) se administra hierro por vía intravenosa $(\mathrm{Fe} \mathrm{IV})^{2}$. Las pautas de administración varían en función de las necesidades de los pacientes, siendo habitual la infusión de $100 \mathrm{mg}$ de Fe sacarosa diluidos en $100 \mathrm{cc}$ de suero fisiológico semanal, quincenal o mensualmente $\mathrm{3}^{3,4,5}$.

Esta pauta de administración se realiza en los últimos 45 minutos de la sesión de HD, no estando establecida la velocidad ideal de perfusión de la dilución. Por ello, nos realizamos la pregunta de si la rapidez en la

\footnotetext{
Correspondencia: Mónica Brazález Tejerina

C/ Angustias 37. 47400

Medina del Campo. Valladolid

E-mail: lospinos@friat.es
}

pauta de administración del hierro intravenoso podría influir en la eficacia del tratamiento, así como en la presentación de efectos adversos ${ }^{6}$.

Para ello nos planteamos los siguientes objetivos:

1. Comparar la eficacia entre una pauta lenta y una pauta más frecuente y ultra lenta de administración de Fe IV, con respecto a los parámetros de la cinética del hierro, así como en los aportes de eritropoyetina y Fe que se realizaron.

2. Analizar si se originan menos efectos adversos por intolerancia con la administración de Fe IV con la pauta ultra lenta.

\section{Material y Métodos}

Se llevó a cabo un estudio prospectivo con 38 pacientes de 2 unidades de hemodiálisis que precisaban tratamiento con Fe IV, siendo cada uno de ellos su propio control. Se compararon diferentes parámetros para los dos tipos de administraciones de Fe IV:

1. LA PAUTA CONVENCIONAL utilizada en ambos centros ( 1 ampolla de $100 \mathrm{mg}$ Fe diluidas en $100 \mathrm{cc}$ de $S F$, que se infundía en los últimos $45^{\circ}$ de la sesión de HD). (Grupo 1). 


\section{PAUTA ULTRALENTA Y MAS FRECUENTE en} la que la dosis anterior se dividía en 3 partes que se administraban equitativamente al paciente a lo largo de toda la sesión de HD (Figura 1). Para un paciente que tenía pautada 1 ampolla de Fe IV semanal se le administró $1 / 3$ de ampolla en cada una de las 3 sesiones semanales. Para el aprovechamiento de 1 ampolla de $100 \mathrm{mg}$ de $\mathrm{Fe}$, esta se diluía en $50 \mathrm{cc}$ de SF; y de esta dilución se obtenían tres dosis para 3 pacientes, que se administraban lo largo de toda la sesión de HD con la bomba de heparina. (Grupo 2).

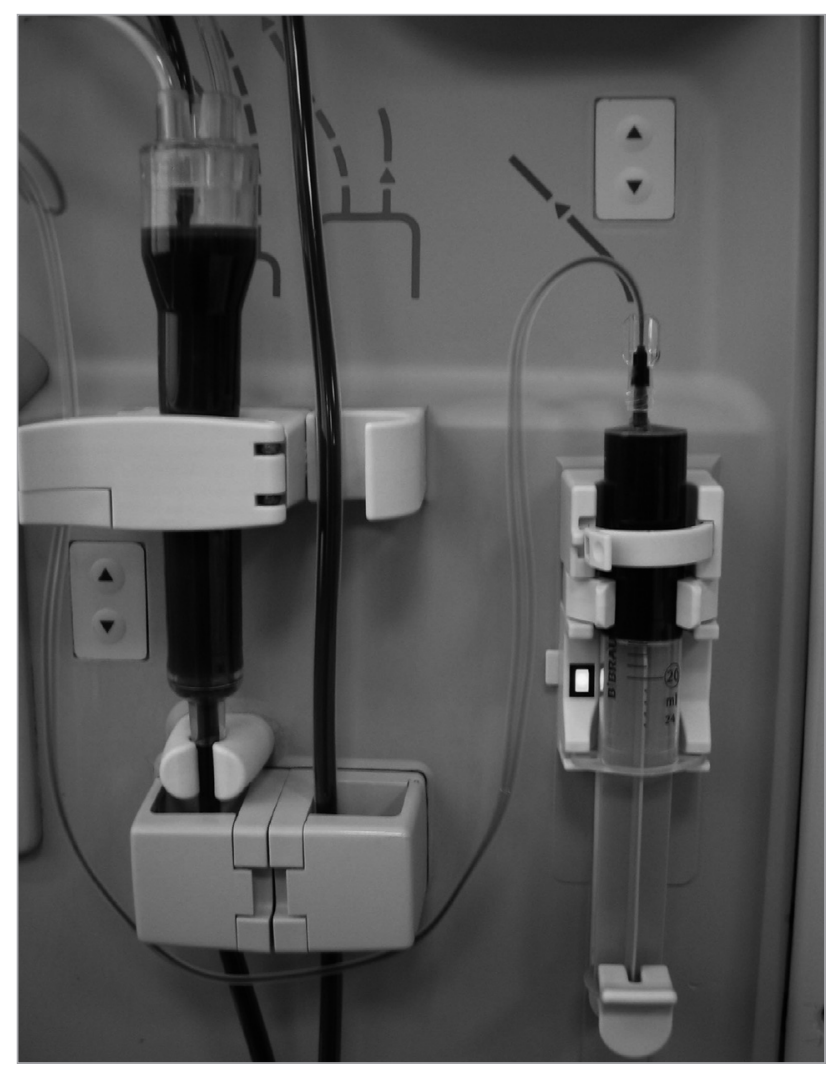

Figura 1. Infusión del hierro intravenoso con la bomba de heparina.
En todos los pacientes se determinó basal y mensualmente: Fe, Ferritina ( Ft), Índice de saturación de transferrina (ISat) y Hemoglobina $(\mathrm{Hb})$. Se valoraron las dosis de Eritropoyetina (EPO) y de Fe mensuales que precisaban los pacientes según los tratamientos prescritos. Y se llevó a cabo un registro exhaustivo de todos los efectos adversos detectados durante la administración del Fe, con ambas pautas de infusión.

El estudio duró 2 años. De Enero a Diciembre del 2008 con la pauta convencional y de Enero a Diciembre del 2009 con la pauta ultra lenta.

Para el análisis estadístico se utilizó la media \pm desviación estándar de la media para la representación de las variables numéricas. Para la comparación de medias entre ambos grupo se utilizó el test de Wilcoxon. Se aceptó significación estadística para $p<0.05$.

\section{Resultados y discusión}

La comparación de medias de los valores de las variables estudiadas al finalizar cada uno de los dos periodos, se muestran en la siguiente tabla.

En efecto, encontramos una disminución tanto de la $\mathrm{Hb}$ y $\mathrm{Fe}$ IV, final vs inicial, que no son significativas, y un incremento de la $\mathrm{Ft}$ y el IST final vs el inicial, igualmente no significativos. Sin embargo, observamos como la dosis de EPO final vs inicial, disminuye de forma significativa $\left(p<0^{\prime} 001\right)$.

La administración intravenosa de hierro sacarosa fue bien tolerada por todos los pacientes en ambos periodos del estudio, no apreciándose efectos adversos durante el tiempo que duró el estudio con las dos formas de perfusión.

\begin{tabular}{|c|c|c|c|}
\hline & PAUTA CONVENCIONAL & PAUTA ULTRALENTA & \\
\hline HIERRO & $73^{\prime} 97 \pm 25^{\prime} 77$ & $73^{\prime} 66 \pm 24^{\prime} 96$ & (n.s.) \\
\hline I.SAT.TRANSFERRINA & $30^{\prime} 26 \pm 10^{\prime} 19$ & $31^{\prime} 21 \pm 99^{\prime} 89$ & (n.s.) \\
\hline FERRITINA & $383^{\prime} 54 \pm 274^{\prime} 87$ & $444^{\prime} 08 \pm 240^{\prime} 44$ & (n.s.) \\
\hline HEMOGLOBINA & $12^{\prime} 47 \pm 1^{\prime} 58$ & $12^{\prime} 13 \pm 1.82$ & (n.s.) \\
\hline DOSIS EPO & $20184 \pm 20064$ & $5763 \pm 4872 ' 34$ & $\left(p<0^{\prime} 001.\right)$ \\
\hline DOSIS HIERRO & $130^{\prime} 26 \pm 84^{\prime} 86$ & $119^{\prime} 08 \pm 83^{\prime} 11$ & (n.s.) \\
\hline
\end{tabular}

Tabla 1. Resultados: comparación de medias 
No surgieron problemas para la aplicación práctica de esta nueva forma de administrar el Fe IV.

\section{Conclusiones}

La pauta ultra lenta y más frecuente de administración de Fe IV consiguió disminuir las necesidades de de Fe IV aunque no de forma significativa. Si embargo, se observó una disminución de la dosis de EPO estadísticamente significativa con esta pauta, al menos en nuestros pacientes. También se observó un aumento de la ferritina y la saturación de la transferrina, aunque no de manera significativa. Para futuras evaluaciones de esta técnica ultra lenta debería tenerse presente otros aspectos relacionados con los accesos vasculares y posibles pérdidas 0 aportes hemáticos.

\section{Bibliografía}

1. Fernández-Gallego J., Ramos B., López de Novales E. Tratamiento con hierro intravenoso e infección en hemodiálisis. Rev. Nefrología 2002; 1 (XXII): 89-90.

2. European best practice guidelines for the management of anemia in patients wich chronic renal failure. Nephrol Dial Transplant 1999. (Suppl. In press).
3. Fernández-Gallego J., Ramos B., Alférez M.J., López de Novales E: Ferritina e infección en hemodiálisis. Evaluación de un protocolo de tratamiento con hierro intravenoso. Nefrología 20: 563-565, 2000.

4. Reyero AM, Marrón B, Manrique MP, Casado S, Ortiz A. Administración mensual de hierro intravenoso en diálisis peritoneal: una pauta eficaz, segura y cómoda. Libro de comunicaciones del XXIV Congreso Nacional de la Sociedad Española de Enfermería Nefrológica 1999; 155-159.

5. Martínez C, Castellanos T, San Vicente J. Aporte endovenoso de hierro en diálisis peritoneal: Una alternativa factible. Libro de comunicaciones del XXV Congreso Nacional de la Sociedad Española de Enfermería Nefrológica 2000; 97-100.

6. Alfaro Cuenca A, Gallego Jordán B, Martín Piñero M, Roca Barrio I, Romero Espinosa E, Sidrach de Cardona García V. ¿Influye la velocidad de administración del hierro sacarosa (Venofer ${ }^{\circledR}$ ) en la ferrocinética del paciente con IRC en hemodiálisis? Libro de comunicaciones del XXIX Congreso Nacional de la Sociedad Española de Enfermería Nefrológica 2004; 188-194. 